\title{
Producción de saberes comunitarios sobre la salud y la lengua guaraní con niños de una comunidad rural de Corrientes, Argentina
}

\author{
Production of community knowledge on health and the \\ Guaraní language in children from a rural community in \\ Corrientes, Argentina
}

DOI: https://doi.org/10.32870/dse.v0i20.597

\section{María Florencia Conde*}

\begin{abstract}
Resumen
El presente artículo ${ }^{1}$ describe los saberes comunitarios sobre la salud y la lengua guaraní que se producen, visibilizan y validan a partir de un proyecto de investigación realizado con niñas, niños y maestras de una escuela primaria rural de la Provincia de Corrientes, Argentina.

El enfoque que atraviesa el estudio es la etnografía en colaboración con niños, desde la cual se considera a estos no solo como interlocutores válidos respecto a los temas de indagación, sino también como productores de conocimientos sobre sus comunidades.

Se pondrán a discusión las relaciones que hemos podido identificar entre las formas de nombrar, describir y usar estos saberes locales, poniendo el foco en aquellos aspectos del proceso de producción que emergen y/o se ponen en debate cuando los mismos se "recrean" desde el espacio escolar. Finalmente, se analizará lo que sucede cuando el aula y el "monte" se convierten en espacios educativos comunitarios en los que tienen lugar otras formas de aprender sobre y en el mundo en el que vivimos.
\end{abstract}

Palabras claves: saberes comunitarios - salud - guaraní - etnografía en colaboración.

\begin{abstract}
This article describes the community knowledge on health and the Guarani language which is produced, visualized and validated based on a research project carried out with children and teachers of a rural primary school in the Province of Corrientes, Argentina.

The study follows an ethnographic approach in collaboration with children in which they are considered not only valid interlocutors regarding questions of inquiry, but also producers of knowledge about their communities.
\end{abstract}

* Profesora en Ciencias de la Educación - Becaria Doctoral UNNE-CONICET del Instituto de Investigaciones en Educación - Facultad de Humanidades UNNE, Argentina.condeflorencia17@hotmail.com

${ }^{1}$ Este artículo está basado en una primera versión elaborada para el V Simposio Internacional de Etnografía con niñas, niños y jóvenes en contextos educativos, organizado por RIEN en octubre de 2017, UNNE-Resistencia, Chaco, Argentina. 
The relationships we identify among the ways of naming, describing and using these local knowledges will be discussed, focusing on those aspects of the production process that emerge and/or are put into debate when they are "recreated" from the school space. Finally, we will analyze what happens when the classroom and the forest become community educational spaces in which other ways of learning, about and in the world we live in will take place.

Keywords: community knowledge - health - Guaraní - ethnography in collaboration.

\section{Introducción}

En el año 2015 llegué a una escuela primaria rural del Departamento de San Luis del Palmar, Corrientes, como parte de un equipo de investigación de la universidad que coordinaría un proyecto de elaboración de materiales didácticos bilingües — guaraní y castellano— sobre hierbas medicinales. ${ }^{2}$ Este material tenía la particularidad de que su contenido sería el producto de una investigación que involucraba a niñas, niños, maestras y directoras de dos escuelas como parte del equipo de investigadores.

Inicié en este proyecto con incipientes preguntas de investigación, participo en un equipo que desde el año 2001 desarrolla estudios sobre los usos y significados del guaraní y el castellano en diferentes contextos escolares y comunitarios de la Provincia de Corrientes. Estos estudios habían partido del discurso de la "prohibición del guaraní", establecido como una ideología lingüística central, para comprender los usos y significaciones del guaraní correntino (Gandulfo, 2007). Dicha prohibición se describe centralmente dirigida a los niños y expresa un conflicto lingüístico que hace que el guaraní se hable, pero tratando a la vez de ocultarlo, o se considere en general que no se habla o se habla poco. "A veces los argumentos para no permitir a los niños hablar el guaraní tienen que ver con que, puedan aprender castellano, 0 , no se les trabe la lengua" (Gandulfo, Miranda, Rodríguez y Soto, 2016:32).

En este contexto, el lugar de la escuela para producir y validar muchas de estas significaciones ha sido clave. Este espacio fue caracterizado como uno de los ámbitos donde la prohibición se hace efectiva de diferentes modos, pero fundamentalmente a partir de la invisibilización de la presencia de niños y maestros bilingües. Sin embargo, estudios más recientes han ido mostrando ciertas tensiones en este "discurso de la prohibición". Si bien actualmente existen prácticas que muestran su vigencia, a su vez se evidencia la vitalidad de la lengua en diferentes ámbitos sociales, así como la emergencia de nuevos usos (Conde y Gandulfo, 2018) y nuevas percepciones acerca del uso del guaraní por parte de los hablantes (Gandulfo, 2016).

\footnotetext{
2 Proyecto de Extensión UNNE en el Medio “Elaboración de Materiales Didácticos Bilingües - guaraní castellano- para nivel primario: las hierbas medicinales y sus usos", dirigido por Carolina Gandulfo, y en colaboración con la Escuela 175, Paraje Albardón Norte y su directora Olga Soto, y con la Escuela 784, Arroyo Pontón cuya directora es Mabel Miranda. Ambas escuelas pertenecientes a la segunda sección del Departamento de San Luis del Palmar, Corrientes.
} 
En el marco de la línea de trabajo del equipo y desde mi participación en el proyecto de elaboración de materiales didácticos, definí como área de interés los saberes locales/comunitarios que se producen, visibilizan y validan a partir de proyectos escolares, haciendo foco en el lugar que ocupa la lengua guaraní, tanto en el desarrollo de estos proyectos como en la producción/ transmisión de estos en la escuela y en la comunidad. A su vez, me interesó describir aquellos aspectos del proceso de producción que se ponen en debate, puja y tensión a la hora de trabajar sobre estos saberes desde el ámbito escolar.

El estudio está atravesado por la etnografía en colaboración como enfoque metodológico, desde el cual se pretende producir conocimiento con la activa participación de quienes son protagonistas de los procesos descritos (Leyva y Speed, 2008). En particular, tienen como referencia los trabajos de investigación en colaboración con niñas y niños, en los cuales se produce conocimiento local a partir de la formación de estos como investigadores nativos de sus comunidades (Podestá, 2009; Milstein, Clemente, Dantas, Guerrero y Higgins, 2011; Gandulfo, 2015). Estos estudios han explorado junto a ellos las formas en que despliegan su vida cotidiana en una diversidad de espacios educativos y sociales, que incluyen el reconocimiento de la agencia de niños y niñas como productores y reproductores activos de prácticas culturales.

Los niños que me acompañan en este trabajo tienen entre 3 y 11 años, viven en el paraje rural Arroyo Pontón, del Departamento de San Luis del Palmar, Corrientes, el cual se encuentra aproximandamente a 35 kilómetros de la capital de la provincia. Todos ellos asisten o asistieron a la Escuela № 784 "Héroe de Malvinas Ramón Cirilo Blanco", lugar en el que nos conocimos y en el cual iniciamos juntos una investigación sobre los usos de las hierbas medicinales, con el fin de elaborar materiales didácticos (Conde y Gandulfo, 2018). Este proyecto se inició en el año 2015 como una propuesta interinstitucional entre dos escuelas primarias rurales y un equipo de investigación de la universidad. El propósito fue formar a los niños de ambas escuelas en las herramientas básicas de la investigación, para que sean ellos los responsables de indagar sobre los conocimientos de sus comunidades respecto a la salud y al uso de hierbas medicinales. Con los resultados de esta indagación se elaboraró el contenido de los materiales bilingües que fueron diseñados y probados por ambas escuelas.

En el marco de las actividades de este proyecto colectivo, ellos han realizado ejercicios de observación en lugares cercanos a la escuela para identificar hierbas con propiedades medicinales; además, diseñaron el instrumento de indagación y entrevistaron a pobladores acerca de los usos de las hierbas, también han analizado todo el material producido y presentaron los resultados en diferentes espacios de divulgación, así como de otras variadas tareas vinculadas a la prueba de los materiales didácticos diseñados. En ambas escuelas se apropiaron de la investigación como una estrategia didáctica para potenciar diferentes competencias de los alumnos. En mi caso, además de compartir los interesés de indagación, me fui enfocando en describir todo lo que implicó el proceso de conformación de un equipo de investigación en la escuela. 
En el año 2017 obtuve una beca doctoral, y a partir de ese momento mis visitas a la escuela ya no tuvieron que ver exclusivamente con las tareas de dicho proyecto. Desde ese año participo de varias situaciones, tanto en la escuela como en la comunidad; la observación participante y las conversaciones son las herramientas fundamentales de producción de la información. Los niños y niñas de este paraje se convirtieron no solo en interlocutores centrales respecto a estos temas sino, también, en compañeros de equipo de investigación con quienes compartimos espacios de producción y discusión respecto a diversas áreas de indagación, algunas propuestas por mí y otras por la escuela.

Como autora de este trabajo, me interesa compartir algunas de las reflexiones que fuimos construyendo en estos años a partir de los diversos encuentros con los niños y adultos de esta comunidad. En los primeros apartados, reconstruyo situaciones en las que los saberes sobre las lenguas y la salud se producen y/o circulan. Enseguida, planteo las relaciones que he podido identificar en las formas de nombrar, describir y valorar estos saberes en la comunidad y, finalmente, analizo lo que sucede cuando el aula y el monte ${ }^{3}$ se convierten en espacios educativos comunitarios.

\section{Saberes sobre las lenguas "del y en guaraní"}

Cuando llegué a la escuela en el año 2015, la mayoría de los adultos y de los niños sostenía que no hablaba guaraní; muy pocos - en general las maestras o algunos padres - expresaban que entendían, pero que les costaba hablar. Los niños generalmente no se reconocían como hablantes, y solo atinaban a expresar sorpresa y timidez cuando me dirigía a ellos usando palabras en guaraní.

Desde que iniciamos el proyecto de materiales didácticos, el guaraní se fue convirtiendo en uno de los temas de conversación más frecuentes, en primer lugar, porque los materiales que nos propusimos elaborar tenían la característica de ser bilingües, pero también por el hecho de que los niños sabían que estaba estudiando guaraní y que tenía un interés particular en hablar de ese tema. La sorpresa me fue invadiendo en diferentes situaciones en las que conversaba con ellos del guaraní y en guaraní, en las que expresaban algunas frases para comprobar cuánto entendía, o bien, en las reflexiones que fueron explicitando respecto al uso de la lengua o a las situaciones de aprendizaje/ transmisión. En general, estas conversaciones tuvieron lugar fuera del aula, aunque progresivamente también fueron emergiendo "adentro".

Uno de los espacios más comunes en el que tenían lugar estas conversaciones era donde está el mástil de la bandera nacional, ubicado justo enfrente de la entrada principal del edificio. Siempre corre una brisa agradable, en verano hay sombra y en invierno da de lleno el sol. Este lugar era uno de mis preferidos durante los recreos, siempre recibía la visita de algún grupo de niños que se acercaba a conversar, jugar, sacarse fotos, etcétera.

3 Forma nativa de designar a un terreno con abundante vegetación sin cultivar.

Diálo@os sobre Educación año 11 | número 20 | enero-junio 2020 | ISSN 2007-2171 
En una de esas tantas ocasiones se acercaron Jazmín, Ezequiel, Karina y Yonibel. Jazmín y Ezequiel llegaron a inicios de 2017 al paraje, ambos nacieron y vivieron en Buenos Aires, ahora viven en Pontón porque su mamá se "juntó"4 con un hombre del lugar. Jazmín es una nena muy cariñosa y demanda atención constantemente. En un momento se sentó a mi lado y me contó que hablaba chino, y a continuación expresó una frase que no logré siquiera transcribir, no la entendí, no solo porque era chino sino porque la dijo muy rápido.

Yo la felicité con un "we, qué bueno Jazmín"y a continuación le pregunté si ya había aprendido guaraní, a lo que ella me respondió "todavía no". En ese momento irrumpieron en la conversación los "yo sí" de Karina y Yonibel, expresando que ellas sí sabían hablar guaraní. A partir de ahí se produjo un breve intercambio sobre las lenguas en las que hablábamos:

FLORENCIA: ¡Ah!, o sea que acá hablamos muchas lenguas. Ella habla chino, yo castellano y guaraní, ¿vos?

KARINA: Guaraní y castellano.

FLORENCIA: ¿Vos?

YONI: : Castellano y guaraní.

FLORENCIA: ¿Y vos?

EZEQUIEL: Castellano.

KARINA: Y cómo es que se dice... porteño ${ }^{6}$ también habla.

EZEQUIEL: Sí.

Luego hice la pregunta de quién les enseñó a hablar esas lenguas. En el caso de Jazmín, ella y su hermano intentaron explicarme que la aprendieron con una amiga china que tenía en Buenos Aires. Luego, me dirigí a Karina, sin especificar a qué lengua me refería, le hice la misma pregunta "¿Y a vos quién te enseñó, Kari?", a lo que me respondió "nadie, escuché por ahí y después aprendí". En el caso de Yoni no hizo falta hacer la pregunta, ella comenzó a contar que en su caso le enseñaba su abuela cuando lavaba las ropas. Después de escuchar la respuesta de Yoni, Karina agregó "cuando hablan mi papá y mi mamá guaraní ahí yo escucho y ahí digo nomás". Enseguida, me miró y me dijo “¡Y a vos?", yo respondí que fue mi abuela la que me enseñó durante la infancia.

La conversación se interrumpió cuando vieron al portero, "don Raúl”y a Mabel —la directora de la escuela - llevar unas cajas. “QQué es eso?”, preguntó Yoni. Yo respondí: “Están por comer algo, parece, jakaru oina [vamos a comer ahora]" Después de las risas por mi respuesta, Karina recordó que su mamá habitualmente dice "oíma la cena" y ella sabe que se refiere a que está

4 Palabra usada por ellos para explicar que su mamá convive con un señor.

5 Palabra usada en Corrientes para expresar sorpresa.

6 Refiriéndose a la variedad del castellano hablada en la Provincia de Buenos Aires. 
lista la comida. Finalmente, la conversación terminó cuando tocó la campana que indicaba la entrada para tomar la merienda.

En otra oportunidad, en el mismo lugar, se acercaron Jordana y Belén. Belén me dijo desde lejos: "Flor, te vamos a hacer unas preguntas, somos investigadoras". Trajeron un cuaderno, una birome y comenzaron con el cuestionario; las preguntas tenían que ver con mi edad, la música que escuchaba, mi comida preferida, etc. En un momento, y como me pareció que finalizaron las preguntas, yo la miré y le pregunté: "ndepa ñe'e guaraní [¿vos hablas guaraní?]", Belén afirmó con la cabeza. A los segundos me volvieron a hacer una pregunta "¿Cómo te llamas?", y yo les volví a preguntar en guaraní "mba'éichapa nde rera [¿cómo es tú nombre?]", Belén me respondió entre risas "Ay, pará que no me salen algunas palabras, o sea... yo entiendo, pero no sé hablar, no me salen las palabras, eso me cuesta, no sé cómo decirte, no me sale de la boca, pero entiendo y te sé responder en mi cabeza". Después de unas cuantas preguntas más se fueron a jugar, prometiendo prepararse para la próxima entrevista.

En estas dos situaciones descritas entraron en juego los saberes sobre las lenguas en general, y el guaraní en particular. Los que estábamos presentes mencionamos las lenguas que hablamos, las usamos y comentamos brevemente cómo las aprendimos, con quiénes y en qué situaciones. Además de una explicitación de las lenguas que usamos, hay una reflexión sobre el uso y las competencias —como es el caso de Belén — que intenta explicar cómo puede entender guaraní sin que le "salga" hablar.

Karina, Yoni y yo comentamos quiénes nos enseñaron a hablar, pero en ningún momento explicitamos que nos estábamos refiriendo al aprendizaje del guaraní y no del castellano. En ese momento parecía obvio, tanto para mí como para ellas, que la pregunta se refería al guaraní; sin embargo, en la lectura de la conversación no queda claro por qué rápidamente asociamos la pregunta con una lengua en particular y no con la otra, o con ambas.

En la conversación de la primera situación, inicialmente no parece haber una jerarquía entre las lenguas y variedades que ellos mencionan "chino, guaraní, castellano y porteño". Yoni y yo ponemos el castellano en primer término cuando mencionamos las lenguas que hablamos; en cambio, Karina ubica primero al guaraní. Esto podría vincularse al hecho de que la lengua "sobre y en" la que más conversamos es el guaraní, y que para tener una participación activa en la conversación es necesario hablary/o entender dicha lengua, pero también puede relacionarse con la percepción que ella misma tiene respecto a sus competencias en ambas lenguas.

Esta situación que describo fue una de las primeras en las que escuché a las nenas explicitar sus posiciones con respecto al guaraní. Esto me produjo conmoción y creo que se debió, por lo menos, a tres razones: en primer lugar, por el discurso que circulaba durante los primeros años en la escuela de que "ahí ya no se habla guaraní"; en segundo lugar, por mi propia dificultad de reconocer que en mi provincia hay niños bilingües; y finalmente, por verme a mí en esas niñas, ver a la Florencia pequeña que no era consciente en su infancia de que su abuela le estaba enseñando una lengua diferente al castellano. 
Allí, justo debajo de la bandera nacional, hablamos del guaraní y en guaraní, es un espacio y momento para eso. Para ellas, ir al mástil a conversar puede tener una variedad de motivaciones e intereses; para mí representa un espacio en el que surgen conversaciones en torno a diversos temas. En dichas conversaciones soy constantemente interpelada a participar como "entrevistada" y no solo haciendo preguntas.

\section{Saberes sobre la salud y el uso de hierbas medicinales Los "aranduminguéra [sabios pequeños]"}

En el proyecto de materiales didácticos los niños tuvieron un papel fundamental, no solo por ser los responsables de realizar el trabajo de campo y analizar la información, sino por el conocimiento que tienen sobre el uso de hierbas medicinales. Esto los ubicaba en el lugar de interlocutores válidos, algo no muy común en las actividades áulicas. Aquí los niños podían discutir con los maestros o entrevistados sobre el uso de hierbas y los procedimientos utilizados en cada caso.

Este conocimiento se hizo evidente desde la primera actividad, cuando tuvimos que definir posibles preguntas e informantes clave. Los niños debían pensar en aquellas personas que — desde sus perspectivas - conocían sobre las hierbas medicinales y se podrían entrevistar. Se armaron largas listas en las que abundaban los abuelos y los "don", lo que demostraba que para ellos los informantes clave eran sobre todo adultos mayores. También se identificaron algunos referentes de las comunidades que curaban ciertas dolencias utilizando hierbas medicinales. Estos eran nombrados como "la médica", "el o la curandera". Ellos mismos establecieron los criterios de selección y definían quiénes sabían "mucho, poco o nada". Las maestras también realizaban sus apreciaciones y en la mayoría de los casos coincidían con la selección de los niños.

También advertimos ese conocimiento en las prácticas de entrevistas entre compañeros, en las que más allá de practicar el uso técnico del equipamiento o el uso del instrumento (objetivo de ese día) se podía observar la capacidad de los niños de sostener una entrevista que fue diseñada para realizar con los adultos. Muchas de las respuestas brindaban ejemplos que eran narrados con mucho detalle, lo que nos hacía suponer que eran situaciones vividas por ellos mismos o alguna persona cercana.

Estos conocimientos acerca de las prácticas de atención a la salud también se explicitaron en conversaciones que fui teniendo con ellos en el marco de mi trabajo de campo durante estos años. Es habitual conversar con ellos sobre sus experiencias o las mías en relación con alguna dolencia en especial y al uso de hierbas medicinales, o bien, sobre las consultas de algunos en el hospital o la salita de alguna "médica o curandera o curandero del paraje".

En una oportunidad, volvíamos con Enrique, Alexis y Ramón de visitar a unos vecinos del paraje. Cuando estábamos llegando a la casa de doña Moró, Enrique dijo, a modo de broma, que pasára-

7 Palabra muy usada en Corrientes. La misma antecede al nombre o apellido de la persona y se usa como expresión de respeto, generalmente hacia adultos mayores. 
mos por esa casa para que la señora nos diera agua bendita. "¿Y por qué tiene agua bendita?", pregunté, "Porque es curandera roncho"8 me respondió Enrique. Mientras Ramón me explicaba

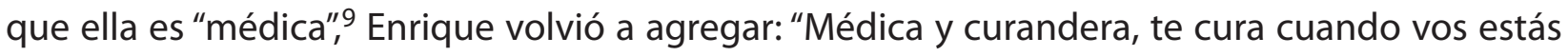
enfermo con la cinta". Enseguida, les pregunté si ellos se habían curado alguna vez con ella, y los tres reaccionaron como si mi pregunta fuera obvia, "Pero... todos los días, más o menos", respondió Ramón. Cuando consulté qué dolencias se curaban con la señora, Enrique me respondió: "los punga" [empachos]. ${ }^{10}$

A su vez, en relación con los procedimientos para curar el empacho, en otra oportunidad Karina me contó que la cinta que se usa en estos casos es como la bandera argentina, azul y en el medio blanca, pero sin el sol. También comentó que es común hacer bendecir la cinta "por la Virgen" en la basílica de la ciudad de Itatí. Además, me explicó que ella a veces va a lo de "doña Moró", como Enrique, pero que en otras oportunidades su mamá la lleva a un "médico que también cura". Este señor le coloca las manos sobre su cabeza, aprieta fuerte mientras reza, y en ese momento Karina cree que "le pasa todo el empacho para él", según ella; nunca entiende lo que dice "porque habla despacito", pero cree que le reza un Padre Nuestro: "No sé bien, porque es como secreto nomá te digo, no se puede saber". Después de ese procedimiento, le indica que tome un té y que coma livianito por unos días.

De este modo, los niños no solo han producido conocimiento sobre estos temas en el marco de un proyecto de la escuela, también han ido recuperando y explicitando sus experiencias al ocupar en variadas ocasiones la posición de "pacientes".

Podría decirse que explicitar que "los niños también saben", supone añadir a la creencia idealizada que ellos tienen un propio mundo, que están por fuera de los discursos y prácticas sociales, que no tienen conciencia de su participación en situaciones que involucran la salud, que no le otorgan sentidos a la misma y que no construyen un saber en torno a los espacios comunitarios que habitan día a día. Sin embargo, creo necesario visibilizar el conocimiento experto que tienen los niños ante estas situaciones, sobre todo porque "los recaudos" que venimos escuchando desde que iniciamos el proyecto de materiales didácticos tienen como principales destinatarios a los niños, a ellos hay que "proteger" de los usos inadecuados de las hierbas o de las negligencias de tratar ciertas dolencias en la casa o de las "prácticas de curanderismo".

Dichos señalamientos de "cuidado" provienen de algunos adultos ajenos al paraje, los cuales al parecer desconocen que estas prácticas comunitarias involucran una serie de componentes y pautas, en los que justamente "los cuidados" " "límites" en los usos de las hierbas ocupan un lugar central. Las prácticas curativas componen un conjunto de conocimientos fac-

8 Palabra usada para expresar que una cosa no lo es completamente, en ese caso, significa "una especie de" curandera.

9 Forma nativa de designar a personas del paraje especializadas en curar algunas dolencias.

10 Indigestión causada por comer en exceso.

Diálo pos 
tuales (perceptivos, gestuales, discursivos, afectivos y sociales) (Gasché, 2010) de los cuales los niños son partícipes, por ejemplo, cuando algunos de ellos o sus allegados son atendidos ante ciertas dolencias, cuando asisten a la curandera para que los cure de los punga, o bien, cuando tienen que buscar ciertos yuyos para la preparación de algún té, entre otras.

Sin embargo, podría decirse que hay un conjunto de conocimientos acerca de las prácticas curativas a las que no todos - tanto adultos como niños - tienen acceso; estos se reservan a ciertas personas que cuentan con el reconocimiento y la legitimidad para hacer uso de ellos. En muchos casos estas prácticas involucran procedimientos secretos que tienen su propia lógica de transmisión (a quiénes se transmiten, en qué momento, etc.). Los niños reconocen que hay personas especializadas, como la/el curandero, que hay dolencias específicas para recurrir a ellos, y que muchos de los procedimientos usados no pueden contarse, como mencionaba Karina con respecto a su experiencia cuando se cura del empacho.

\section{Piedras en el camino: las interconsultas}

El año en el que empecé a frecuentar más la escuela sufrí un cólico renal. Después de los estudios correspondientes me identificaron cuatro piedras, dos en el riñón izquierdo, una en el derecho y otra en el conducto de la vejiga; esta última fue la que me había producido el cólico.

Lo primero que hice fue ir al urólogo, quien me recetó unas pastillas para ayudar a expulsar la que estaba ubicada en el conducto de la vejiga; si no lograba expulsarla, tendrían que realizar un procedimiento quirúrgico para retirármela. La contraindicación de este medicamento era que me podría producir presión baja, lo que no me agradaba en absoluto. Ante las indicaciones del doctor, le consulté si el "rompepiedras"11 — del cual me habían hablado en el paraje — me podría ayudar. Recuerdo que me miró, dudó unos segundos y después me respondió: “Mira, yo no te lo puedo indicar porque soy médico y no está avalado por las ciencias médicas, pero he visto milagros".

Durante esa semana estuve con muchas dudas con respecto a qué hacer, estaba mejor, ya no sentía el dolor intenso de la semana anterior. En mis visitas a la escuela consultaba a todos los que podía sobre la efectividad del rompepiedras. Aproveché una reunión de padres en la que hablamos - entre otras cosas - del proyecto de materiales sobre hierbas medicinales. En la conversación sobre las hierbas, doña Lidia12 expresó: "Algunos pronto no quieren tomar porque no creen, tenés que creer para que te haga efecto". Mabel, la directora, les contó a las mamás presentes sobre mi "problema"; todas ellas me dijeron que tomara el rompepiedras, que me iba a hacer bien, algunas de ellas incluso se ofrecieron a mandármelo el día siguiente.

La semana siguiente Belén me llevó una bolsita con rompepiedras; cuando me la entregó, me recomendó no preparar el té muy fuerte y no ponerle azúcar para que me hiciera efecto. Yo

11 Planta conocida por sus efectos para disolver cálculos en los riñones o la vesícula.

12 Doña Lidia es una tutora muy presente en la escuela, es mamá de tres chicos y abuela de uno, además de otros cuatro que ya egresaron. 
le consulté si podía hervirlo, dejarlo enfriar y tomar como agua fría. Ella me respondió: "Toma como quieras, frío, caliente, pero no dejes de tomar". A partir de ese momento, Belén se convirtió en una especie de enfermera, no solo me llevaba rompepiedras casi todas las semanas, sino también se encargaba de controlar si tomaba lo suficiente y de reprocharme si le decía que no me cuidaba.

Dos semanas después de esa reunión de tutores, realizamos una actividad en el monte lindante a la casa de doña Lidia, de la misma participaron algunos niños, cuatro mamás, la directora de la escuela y la directora del proyecto de materiales didácticos. Esa mañana hizo mucho calor, yo no me había recuperado completamente de los cólicos, tenía molestias y por momentos me bajaba la presión. Durante la actividad estuve bien; sin embargo, al volver a la casa de doña Lidia — pleno mediodía a 40 grados- empecé a sentir mareos y nauseas.

En la casa de doña Lidia, su hija había organizado el almuerzo para todos en el patio de la casa, yo me senté aparte y dije que iba a esperar para comer porque estaba un poco descompuesta. Todos los presentes hacían chistes con respecto a mi descompostura; sin embargo, doña Lidia se mostró preocupada, dejó de comer, me hizo apoyar las piernas en una silla y se acercó a mí con una botella de agua bendita. Comenzó a mojarme la cabeza mientras cerraba los ojos y susurraba unas palabras, mezclando guaraní y castellano. Yo no lograba entender qué decía, solo escuchaba recurrentemente la palabra ñandejara (Dios). Esto duró aproximadamente cinco minutos, ella estaba convencida de que se trataba del hígado o de la vesícula, por eso me dio un té de boldo en saquito, de esos que se compran en los negocios, y me buscó rompepiedras de su patio para que me preparara un té al llegar a casa. Cuando terminó me dijo que había hecho una bendición y que ya me iba a empezar a sentir mejor.

De a poco comencé a estabilizarme, en ese momento no podía entender qué me estaba haciendo. Solo recordaba las situaciones de mi infancia en las que asistía a un señor para que me curara los cadillos, o bien, en las que visitaba a mi tía para que me "mida" el empacho, o aquellas situaciones actuales en las que mando por mensaje de texto mi nombre a una señora para que esta me cure a la distancia de la descompostura del estómago o del ojeo. En todas estas situaciones, mi convencimiento y confianza en cuanto al procedimiento no estaban en duda; sin embargo, en el procedimiento de doña Lidia experimentaba la sensación de que tenía que entender todo lo que estaba pasando en esa situación y, a la vez, esa pretensión de entender me llevaba a desconcentrarme de lo que me estaba pasado a mí y a mi cuerpo, allí, en ese mismo momento.

Durante los meses que siguieron no volví a tener molestias en los riñones, tomaba mucha agua, el rompepiedras — aunque no con la constancia que me indicó Belén- y había recibido la bendición de doña Lidia. Después de un tiempo volví al doctor, la piedra de la vejiga ya no estaba; sin embargo, la del otro riñón aumentó de tamaño y tenía una nueva del lado derecho. Ante esto, volví a tomar el rompepiedras durante dos semanas y logré expulsar, casi sin dolor, al menos una piedra más. 
Al momento de escribir este artículo me encuentro en un proceso de toma de decisión respecto a la piedra que aún se encuentra alojada en mi riñón, consultando nuevamente a mi doctor de la clínica y a mis "doctoras" del paraje (doña Moró, doña Lidia, Belén) buscando además algunos consejos para prevenir la aparición de otras nuevas.

En este apartado me pongo a mí y a mi problema de salud en el centro de la escena. En ese momento, además del dolor, sentía mucha contradicción con respecto a la decisión que tenía que tomar, si recurrir a la medicina de la clínica o creer en la medicina del paraje, que además era el tema de indagación en ese momento. Si bien el doctor señaló que "había visto milagros", yo no terminaba de confiar, y eso era clave, tal como lo expresa Doña Lidia, para que "haga efecto". Creo que mi incredulidad se manifestaba especialmente en no seguir la indicación de Belén de que fuera constante, no era rigurosa ni sistemática para consumir el rompepiedras, como tal vez sí lo soy cuando el doctor me receta algún medicamento.

En cierto sentido, la necesidad de que la gente me asegure que el rompepiedras iba a solucionar mi problema de salud tenía por debajo la duda. Pero esta desconfianza no se debía al efecto en sí mismo, sino justamente a lo que expresó doña Lidia con respecto a "creer", yo dudaba de que me hiciera efecto, no por la acción en sí misma de la hierba sino porque yo no terminaba de creer.Y según lo que venía escuchando, y dadas mis propias experiencias frente a otras prácticas de curación, la "creencia" del paciente es un requisito fundamental para el éxito del procedimiento. En este caso, yo experimentaba muchas dudas y no quería volver a atravesar por un cólico.

Mi descompostura en la casa de doña Lidia y su intención de colaborar para que me sintiera mejor fueron claves para tener confianza, no sé bien en qué consistió "la bendición", no sé bien qué dijo, pero observé la seriedad con la que llevó adelante el procedimiento. Creí en su palabra, en su experiencia y en sus buenas intenciones. Y más allá de la confianza, o por la confianza, efectivamente me sentí mejor. La incomprensión de aquella situación persiste; sin embargo, la lectura de la antropóloga Favret Saada (Zapata y Genovesi, 2013) me brindó algunas pistas para pensar esa escena. Con esta autora — salvando las enormes distancias de nuestros trabajos - pude entender esta situación como parte de la construcción de mi objeto de conocimiento. En primer lugar, la cuestión de "aceptar dejarse afectar" —en su caso por la brujería en la Francia contemporánea-, lo cual, según la autora, no se trata ni de la observación participante, ni mucho menos de empatía.

Ya que las posiciones se ocupan en lugar de imaginarlas, por la sencilla razón de que lo que ocurre en su interior es literalmente inimaginable [...] Ocupar esas posiciones no me informa nada sobre los afectos del otro; ocupar tal lugar me afecta, es decir, moviliza o modifica mi propio bagaje de imágenes (Zapata y Genovesi, 2013:63).

Por su parte, la antropóloga Ramírez Hita (2009) destaca los aportes que nos brinda el enfoque etnográfico para abordar los temas vinculados a la salud, en su caso, la realidad sanitaria de la ciudad 
de Potosí, Bolivia. En particular, pone en consideración la percepción de los datos mediante el cuerpo del investigador, para comprobar cómo cambia radicalmente la relación que se mantiene con los sujetos o con el grupo de estudio cuando uno efectúa la misma actividad que los "otros". Esta autora, mientras realizaba trabajo de campo sobre la calidad de atención en los hospitales públicos del Altiplano, fue internada en un hospital para ser intervenida quirúrgicamente. A partir de esa internación, los datos obtenidos previamente mediante las técnicas de entrevista y observación cobraron un sentido radicalmente diferente. De este modo, poner su cuerpo en acción, entendido como "el cuerpo situado en el sentir" o "el cuerpo sintiendo", le proporcionó un nivel de percepción del dato, que va mucho más allá de la información que cabe recoger por medio de las técnicas de metodología cualitativa que había utilizado anteriormente (Ramírez Hita, 2009).

Si bien la lectura de estos textos me permitió poner en foco mi experiencia en relación con las cuestiones que estaba indagando, considero necesario atender a uno de los puntos destacados por Favret Saada, quien expresa que "en el instante en el que uno está afectado no puede relatar la experiencia; cuando se la narra, no es posible comprenderla. El tiempo para el análisis viene después" (Zapata y Genovesi, 2013:63). En mi caso, entiendo que todavía tengo que tomar muchas decisiones con respecto a mi problema de salud y a la gente que me está ayudando a tratarla, intentando - como hizo esta autora — en ningún momento resignarme a no comprenderla, y a considerarla como parte de mi objeto de investigación.

\section{Cuando los saberes se encuentran... En el aula}

Como parte del proyecto de materiales didácticos, se organizaron actividades de análisis de las entrevistas que los niños habían realizado. Mabel coordinaba el grupo de jardín y el primer ciclo. Allí participaban los nenes, las dos maestras, Graciela —mamá de Brian, de jardín-y don Raúl. Yo me encargaba de coordinar el grupo de segundo ciclo. Habíamos llevado unas computadoras y unos cuadros impresos que ellos debían completar.

Desde el inicio del proyecto, las maestras mostraron el conocimiento que tienen con respecto al uso de las hierbas, muchas de ellas tenían su propia "farmacia de yuyos" en sus casas y era común escucharlas comentar sobre los usos habituales que hacen para determinadas dolencias. En ocasiones, esto hacía que juzgaran las respuestas de los entrevistados, que quieran corregirlas, incluso una seño ${ }^{13}$ se compró un libro de hierbas durante el proyecto, el cual propuso usar de referencia, sin considerar que lo central estaba en el conocimiento que habían producido los niños.

Durante la actividad de análisis, yo acompañaba más de cerca a un grupo de varones que hacían constantemente preguntas sobre cómo completarla, todos estaban entusiasmados, in-

13 Modo de nombrar a las maestras.

Diálo@os 
cluso Lucas, uno de los nenes más grandes, que habitualmente me confrontaba, quejándose de cada tarea que realizábamos. Siempre participaba, pero parecía estar todo el tiempo enojado. Cuando explicaba algo, generalmente me hacía burlas o me cuestionaba lo que decía. Ese día estaba muy concentrado junto con sus compañeros, yo me alegré porque lo vi sonriente todo el tiempo, salvo cuando me acercaba a preguntarle alguna cuestión vinculada a la actividad. En una de esas oportunidades, me dirigí a él felicitándolo porque había terminado su planilla; él me miró con un gesto de desinterés y me preguntó: "¿Y esto para que nos sirve?". No era la primera vez que Lucas me dejaba unos segundos en silencio, siempre me desafiaba a correrlo del lugar de "niño" y responder a sus preguntas o comentarios con la misma seriedad que tendría ante un adulto. Le expliqué que lo que estaban haciendo era un ejercicio de análisis y que, más allá de aprender a hacer cosas nuevas que les pueden servir para otras tareas, también estaban haciendo una actividad para un proyecto que involucraba a toda la escuela. "¿No te gusta?", le pregunté. "No, ni ahí", me respondió. Yo le sugerí que hablara con la directora para hacer otra actividad. Sin embargo, Lucas fue el primero en terminar. Según las maestras "él siempre se queja, pero hace" y según sus compañeros, "es siempre mala onda". Yo agregaría a esos comentarios que "siempre hace preguntas interesantes" como esta o como la que hizo durante una de las prácticas de entrevista a las que inicialmente se resistió. Ese día preguntó: "¿Qué estamos aprendiendo con esto?".

Fotografía 1. Niña realizando una actividad sobre el uso de hierbas medicinale

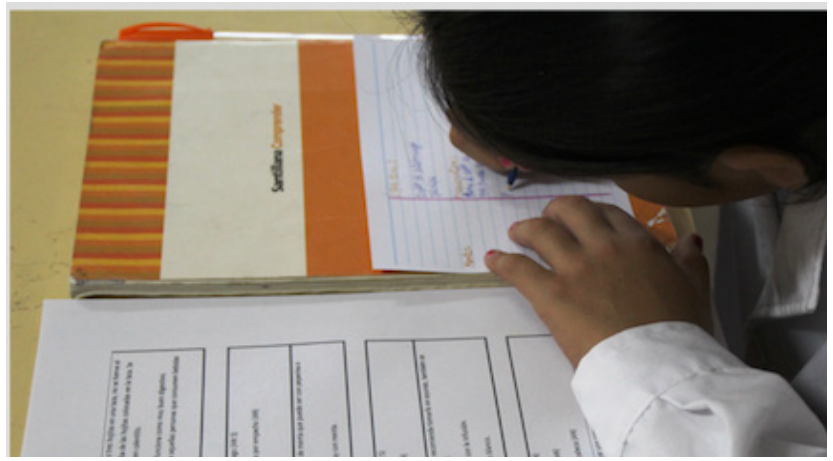

En esta actividad de análisis, como yo me encargaba de acompañar a los varones, la seño de sexto grado guiaba a las niñas. Su participación era prácticamente nula, ellas habían entendido la consigna y se pusieron a trabajar inmediatamente; sin embargo, en algunas ocasiones recurrían a ella. En un momento de la actividad, Guada le comentó que una entrevistada había dicho que la ruda es para las malas ondas. Ante esto, ella respondió que sí, que así dicen, pero que eso no lo iban a poner. Cuando escuché ese comentario, decidí acercarme y preguntar por qué no iban a incluirlo. Ella me miró con cara de duda y me respondió "bueno, pongamos... yo decía porque no es algo comprobado". Unos meses después de esa actividad, esa misma seño contó en una conver- 
sación con las demás maestras, que su ruda estaba seca, que andaba circulando mala energía en su casa. Al parecer, usaba la ruda con los mismos objetivos que la entrevistada.

Otra actividad de análisis fue la confección de lo que en la escuela llamaron el "Glosario guaraní-castellano", aunque no se definieron las palabras, sino que se buscó su traducción del guaraní al castellano. La actividad la inició Mabel. A pesar de declarar una escasa competencia lingüística en guaraní, pudo proponer y llevarla adelante obteniendo mucha participación de los niños. Esta actividad también la realizó la maestra de tercer grado con sus alumnos, en este caso ella participó activamente ya que, según sus dichos, "entiende bastante el guaraní". Finalmente, las producciones quedaron plasmadas en afiches que fueron pegados en las paredes del aula.

Este "glosario" fue retomado luego por una de las maestras como un punto de la evaluación trimestral de Lengua, en la que —según comentó- tuvo excelentes resultados dado que los niños no tuvieron errores al responder ese punto. La docente expresó su asombro al comprobar cuánto sabían, "sabían muchísimo, saben más que yo. Siempre escribiendo como hablamos ¿no?, no el guaraní puro, digamos." Esta maestra era la misma que sugirió no poner las propiedades de la ruda en el trabajo.

En este apartado, los saberes sobre las hierbas y el guaraní se producen y circulan en el aula, en actividades de las que participan no solo docentes y alumnos, como habitualmente sucede. Se organizaron equipos conformados además por la directora, el portero de la escuela, una madre y yo como "becaria". A pesar de que la mayoría de los presentes tenían conocimientos sobre las propiedades medicinales de las plantas de la zona, lo cierto es que los niños eran los que más familiarizados estaban con el material empírico ya que fueron ellos los encargados de hacer las entrevistas.

Esto último marca una diferencia en relación con otros tipos de saberes que generalmente se explican, enseñan, aprenden, o no, en ese espacio, los cuales en general son exclusividad de los docentes. Ese día las maestras podían aportar, pero los expertos eran otros actores (don Raúl, Graciela) y los propios niños, quienes además discutían sobre la pertinencia de determinadas respuestas.

El criterio de qué información incluir en el trabajo, pareciera ser —según la perspectiva de una de las maestras- que ese conocimiento esté comprobado. Su criterio tiene cierta justificación considerando que, por ejemplo, si hay dolor de panza, se toma un té de alguna hierba y en general se experimenta una mejoría. ¿Cómo se hace en el caso de las malas ondas? ¿Cómo se experimenta un alivio? En su cotidianeidad se usa la ruda para las malas energías y tiene como indicador si está se seca o no. Ahora bien, su experiencia "comprobada" no pareció suficiente como para incluir esa respuesta en un trabajo de investigación realizado en el aula.

Sin embargo, en otro momento, los saberes de los niños sobre el guaraní son puestos en valor por esa misma maestra, y no solamente eso, sino que son considerados en una evaluación trimestral de Lengua. No es un dato menor que la evaluación haya sido la de Lengua, en la que 
generalmente los chicos tienen mayores dificultades. Las maestras hacen un importante esfuerzo para que hablen y escriban "bien", es decir, sin errores ortográficos, sin marcas del guaraní ni de la variedad del castellano hablado en Corrientes. La decisión que se tomó implicó en cierto sentido un desafío ya que se trató de incluir, validar y evaluar palabras escritas según "como hablamos", es decir, no en guaraní "puro", tal como ella aclaró en un momento.

En este sentido, que el guaraní aparezca en una actividad de mucha importancia en la escuela, como son las evaluaciones trimestrales, y no solo en otras situaciones en la que se corre el riego de folclorizarlo, lo posiciona en un estatus similar al que ocupa la variedad estándar del castellano, desde la que habitualmente se pretende evaluar en estas instancias.

Las dudas con respecto a la confiabilidad del conocimiento que se estaba produciendo se manifiestan, por ejemplo, cuando una de las maestras se compra un libro de hierbas para consultar o chequear la información brindada por los entrevistados. El hecho de que sea un libro podría hacer suponer que cuenta con una validez científica per se. Las dudas también aparecían en el momento de comunicar este conocimiento ya que en el intercambio parecía estar bien comentar, por ejemplo, sobre los usos de la ruda para las malas ondas; sin embargo, no correspondía al momento de volcar esa respuesta en una planilla, como parte de las actividades de la investigación. En ese momento, operaba el criterio de que este conocimiento "esté comprobado", no por la propia experiencia efectiva de la docente sino por criterios que tienen otra legitimidad.

Finalmente, la duda también se expresa en las preguntas de Lucas, el "¿para qué nos sirve?" y el "¿qué estamos aprendiendo con esto?", pueden ser parte de los cuestionamientos que realiza habitualmente, de su interés genuino de entender el para qué, pero también podría expresar una cuestión más profunda, que tiene que ver con la pregunta ¿por qué hablar de estos temas en la escuela?

\section{En el monte}

Otra de las actividades del proyecto de materiales didácticos consistió en una salida al monte ${ }^{14}$ para fotografiar las hierbas medicinales que habían sido mencionadas en las entrevistas realizadas por los niños a los adultos de la comunidad. El lugar elegido fue el monte lindante a la casa de doña Lidia. De la salida participaron algunos niños - ya que la actividad no era obligatoria-, cuatro mamás, la directora de la escuela, la directora del proyecto de materiales didácticos y la autora de este trabajo. Fue una mañana de mucho calor. Cuando llegamos a la casa, doña Lidia nos esperaba lista con su machete para empezar el recorrido. Su propósito era llevarnos "monte adentro" y lo primero que resaltó fue que nuestra ropa (la mía y la de la directora del proyecto y de la escuela) no era la apropiada para el lugar donde íbamos. Algunos de los nenes, en cambio, estaban con gorras, camisas de manga larga y calzados más apropiados que los nuestros.

14 Brevemente descrita en el apartado "Piedras en el camino".

Diólo@os 
El monte al que entramos era muy tupido y constantemente necesitábamos que doña Lidia "nos abriera camino" con su machete, a la vez que nos iba explicando las propiedades de las diferentes plantas con las que nos encontrábamos en el camino; ella era la que daba la explicación central, mientras que las otras mamás comentaban algunos ejemplos de uso de las hierbas. En muchos momentos la explicación se dirigía centralmente a mí, "para mi trabajo", ya que yo era la encargada de registrar en un cuaderno las plantas que íbamos encontrando. Los nenes que nos acompañaban seguían el recorrido sin agregar mucho, pero algunos de ellos — sobre todo los hijos de doña Lidia - podían reconocer las plantas de las que hablábamos, señalando dónde estaban o, incluso, buscando ellos mismos algunas que no encontrábamos a simple vista. También reconocían cuáles eran las peligrosas o las que ellos no pueden consumir, o bien hacían constantes referencia a "lo amarga o fuerte" que eran algunas de ellas.

Ese día, además del conocimiento que tenían los chicos sobre los usos medicinales de las hierbas -lo que ya había advertido en otras ocasiones-, lo que llamaba mi atención era el conocimiento que tenían sobre cómo "estar" en el monte, las dinámicas de participación que se establecían, en las que muchas de las actividades estaban restringidas a los varones mayores (uso del machete, confirmación de que se trata de la hierba que buscábamos, separar a los perros cuando se peleaban, etc.), el rol de los más grandes para "guiar" a los más chicos, las conversaciones que giraban en torno a lo que íbamos encontrando, a las situaciones que se nos presentaban (cruzar un charco, las altas temperaturas que nos acompañaban) o a las experiencias en cuanto al uso de alguna hierba específica.

\section{Fotografía 2. Niños buscando una hierba medicinal en el monte}

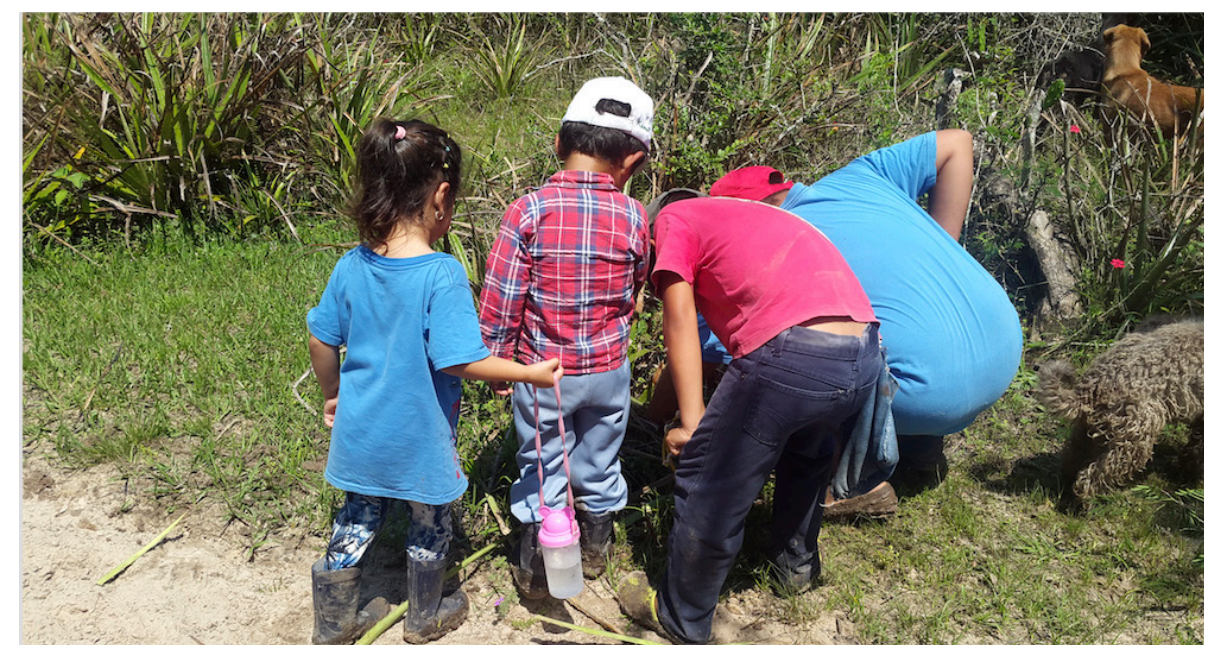

En un momento de ese recorrido nos encontramos con la planta mío-mío, Belén nos advirtió que era una planta peligrosa, lo que fue confirmado por doña Lidia, quien expresó que 
"te mata directo", recordando la historia de un anciano que se hizo un té porque "tenía una pere" [herida]. En ese momento pregunté en voz alta si pere era "herida" en guaraní. Gustavo, que venía a mi lado, me respondió que significaba "que está cortado". Belén escuchó la respuesta y expresó "y sí, él habla todo el día en guaraní, le habla a la seño en guaraní para que no entienda, pero yo sí entiendo". A partir de ese momento, Belén me fue contando la experiencia de su familia con respecto al uso de esta lengua, volvió a resaltar — como en la conversación junto al mástil— que ella entiende, pero que no le sale bien, que su mamá entiende un poquito, que escucha pero que tampoco le sale, y que su papá lo habla desde chiquito. También me contó que, a veces, para practicar le dice a su abuela: "Abuelita, decime algo en guaraní para ver si está bien o está mal", y que ella se da cuenta de que algunas palabras ya no se encuentran más en guaraní, como tomate, por ejemplo "ya no es más guaraní, se inventan nomás, no es como mba'e [qué], ese ya es verdadero".

Gustavo escuchaba la conversación en silencio; sin embargo, no dejó de hablar guaraní a medida que avanzaba la caminata, las frases que expresaba - y que yo lograba escuchar o entender- tenían que ver con las situaciones del momento haku kuarahy [sol caliente], nde karapengo [sos petiza], refiriéndose a mí altura al momento de soltar una rama con espinas, oñerei [hablan de balde] cuando conversábamos con Belén de los miedos que le tenemos a ciertos animales; o bien cuando se refería a una característica de las plantas con las que nos encontrábamos, por ejemplo, que eran ácidas (refiriéndose al aguahai) o especificando que el llantén ${ }^{15}$ es para el jai [diente] de los más pequeños.

Al escuchar nuestros intercambios, las mamás que nos acompañaban recordaron anécdotas de uso en guaraní en la ciudad de Corrientes, ante situaciones en las que descubren sorprendidas que las otras personas las entienden, y reconstruyendo en guaraní los diálogos que tuvieron en ese momento, sin sentir la necesidad de traducir a los que estábamos presentes.

Allí, en medio del monte, todos intercambiamos hablando el guaraní, desde doña Lidia como la más experta de la situación —-mezclando guaraní y castellano durante todo el recorrido—, hasta lgnacio (de 3 años), el más pequeño del grupo, gritando teju [iguana] al ver que los perros habían cazado este animal. No era la primera vez que participaba de situaciones en las que el guaraní era el tema de conversación; sin embargo, acá no solo hablamos del guaraní, sino que se usaba como parte de las situaciones que nos iban pasando en el recorrido. Los saberes sobre la lengua y sobre las hierbas medicinales emergían como parte de reportorios de conocimientos que los presentes producíamos y usábamos para "estar" en el monte.

\section{Reflexiones finales}

En este ensayo reconstruyo situaciones de trabajo de campo en las que los saberes se producen y/o entran en juego, intentado describir quiénes son los actores que los encarnan, los espacios en

15 Planta que se usa con fines medicinales. 
los cuales circulan y algunas de las tensiones que emergen cuando estos pretenden ser legitimados en el espacio escolar.

En estos años de trabajo en la escuela y en la comunidad fui identificando características similares en las formas de nombrar, describir y usar los saberes sobre las hierbas medicinales y los saberes sobre el guaraní.

Una de ellas tiene que ver con la no explicitación en un primer momento de ese conocimiento, no se dice que se sabe o se cree que se sabe muy poco. El tiempo y la interacción en diferentes circunstancias, espacios y con diferentes actores posibilitó que estos conocimientos pudieran ser explicitados, o bien, "usados" con una finalidad específica, para hacer una actividad escolar o para "estar" en el monte. A su vez, estos saberes fueron legitimados desde la escuela por ser necesarios para concretar un proyecto escolar. En este sentido, todo lo que "saben" los niños, no solo fue una sorpresa para mí, para ellos inicialmente también resultaba difícil dimensionar cuánto sabían sobre estos temas, hasta que se fueron encontrando con situaciones que implicaban explicitar estos saberes para concretar actividades específicas.

Otra característica identificada tiene que ver con el lugar de los abuelos "los antiguos" - como ellos los definen - en la enseñanza y/o transmisión de estos conocimientos. A su vez, hay una diferenciación de generaciones "que saben más que otras" y generalmente existe la creencia de que las generaciones de los hijos/nietos son las que "saben menos". En las diversas actividades del proyecto estuvo presente la consideración de que los "antiguos saben más"; sin embargo, también fue central el conocimiento que los propios niños tenían respecto a estos temas. En este sentido, se puede discutir no solo las posiciones que los sobreprotegen, sino también aquellas que exotizan el uso de hierbas medicinales, negando que se trata de conocimientos cotidianos que se adquieren desde temprana edad.

Por último, es común escuchar la creencia expresada por los actores de que no poseen el conocimiento "puro", en el caso de la lengua, o "científico", en el caso de las hierbas medicinales. En ambos casos ubican estos conocimientos en un estatus diferente a los saberes hegemónicos sobre la salud y las lenguas.

Cuando comencé a pensar las relaciones entre estos saberes tenía varios supuestos, uno de ellos era la idea de que los conocimientos locales sobre los usos de hierbas medicinales y ciertos procedimientos de curación - como los casos de empacho, ojeo- se trasmitían mayormente en guaraní. Tenía una visión un poco idealizada de esta relación, ya que pareciera que mi expectativa era que ahí, "justo" en ese momento, iba a escuchar a las personas hablar guaraní, algo no muy habitual para mí cuando recién comenzaba el trabajo de campo.

Sin embargo, en este tiempo de trabajo observé que la gente no usa el guaraní exclusivamente en estos momentos y con esos temas; a veces sí, a veces lo mezclan, y en otras ocasiones usan mayoritariamente castellano. En este sentido, pareciera que no consideré que los saberes sobre la salud y el uso de hierbas no siempre son verbalizados, y mucho menos 
aprendidos interrogando a la gente. En gran medida se trata de saberes-hacer que están articulados con el funcionamiento de las prácticas sociales (Gasché, 2010) y que se aprenden a partir de la participación en comunidades de prácticas (Lave y Wagner, 1991). Estos saberes-hacer involucran diferentes actores y tienen lugar en una diversidad de contextos de aprendizajes, en el caso de los niños, al ocupar la posición de "pacientes" o siendo partícipes de situaciones en las que algún allegado presenta alguna dolencia/enfermedad, o bien, como en este caso, indagando sobre estas temáticas para una actividad de la escuela.

En este marco, resulta difícil pensar los usos del guaraní y el castellano como exclusivos de espacios estáticos o de esferas de conocimientos cerrados; se trataría más bien de procesos que pueden estar vinculados a la situación sociolingüística de la Provincia de Corrientes, la cual está lejos de ser comprendida desde perspectivas monolíticas.

Entonces, pareciera más pertinente apelar a otras formas de analizar las relaciones entre estos conocimientos considerando, por ejemplo, el monte como un espacio educativo comunitario en el que se producen, trasmiten y articulan dichos saberes. Esto supondría pensar desde otros modos y lógicas de aprender sobre y en el mundo en el que vivimos.

No obstante, también podría suponer una limitación el hecho de considerar que solo en el monte es posible que estos saberes se "encuentren". El proyecto de investigación para elaborar los materiales didácticos posibilitó "hacer monte" en la escuela, es decir, crear espacios educativos comunitarios que recrean la vida social, lo cual supuso que tengan lugar otras dinámicas de interacción, que los niños recuperen sus propias experiencias de vida, las actualicen y legitimen en el marco de la investigación, que aprendan con y de otros niños y adultos, que se apropien de sus roles como productores de conocimiento local y que, finalmente, se pueda poner en tensión la hegemonía de ciertos saberes considerados socialmente válidos.

\section{Referencias}

Conde, M. y C. Gandulfo (2018). Nuevos usos y significaciones del guaraní en un proyecto de elaboración de materiales didácticos en Corrientes. La Rivada, 6(10). Universidad Nacional de Misiones. http://www.larivada.com.ar/attachments/article/176/dossier conde gandulfo la\%20rivada\%2010.pdf

Gandulfo, C. (2007). Entiendo pero no hablo. El guaraní "acorrentinado" en la escuela rural: usos y significaciones. Buenos Aires: Editorial Antropofagia. http://pdfhumanidades.com/sites/ default/files/apuntes/Gandulfo\%2C\%20leer\%20cap\%201-3.pdf

(2016). Hablan poco guaraní, saben mucho: Una investigación en colaboración con niños y maestros en un contexto bilingüe de Corrientes, Argentina. Signo y Seña, 29, 79-102. http://revistas.filo.uba.ar/index.php/sys/article/view/553 
M., M. Miranda Rodríguez y O. Soto (2016). El guaraní correntino. En: Quichua y guaraní: voces y silencias bilingües en Santiago del Estero y Corrientes, 15. Colección Pueblos Indígenas en la Argentina: historias, culturas, lenguas y educación. Buenos Aires: Ministerio de Educación y Deportes de la Nación. http://www.bnm.me.gov.ar/giga1/documentos/EL005246. pdf

Gasché, J. (2010). Qué son "saberes" o "conocimientos" indígenas, y qué hay que entender por "diálogo"? En C. Pérez y J.A. Echeverri (eds.). Memorias Primer encuentro amanzónico de experiencias de diálogo de saberes: Leticia, 10 al 12 de noviembre de 2008. Colombia: Editorial Universidad Nacional de Colombia Sede Amazonia. http://www.unter.org.ar/imagenes/ Memorias\%20Dialogo\%20Saberes\%20VER\%20GASCH\%C3\%89.pdf

Lave, J. y E. Wenger (1991). Situated learning: legitimate peripheral participation. Nueva York: Cambridge University Press. http://www.sciepub.com/reference/40404

Leyva, X. y S. Speed (2008). Hacia la investigación descolonizada: nuestra experiencia de co-labor. En: X. Leyva, A. Burguete y S. Speed (coords.). Gobernar (en) la diversidad: experiencias indígenas desde América Latina. Hacia la investigación de co-labor (pp. 34-57). México: CIESAS/FLACSO-Ecuador/FLACSO-Guatemala. http://files.matices-ugc.webnode. es/200000046-65559674bf/Leyva y Speed.pdf

Milstein, D., A. Clemente, M. Dantas, A. Guerrero y M. Higgins (2011). Encuentros etnográficos con niñ@s y adolescentes. Entre espacios y tiempos compartidos. Buenos Aires: Miño y Dávila.

Podestá, R. y niñas, niños nahuas y de la ciudad (2008). Encuentro de miradas. El territorio visto por diversos autores. México: Coordinación General de Investigación Intercultural-SEP.

Ramírez-Hita, S. (2009) La contribución del método etnográfico al registro del dato epidemiológico. Epidemiología sociocultural indígena quechua de la ciudad de Potosí. Salud Colectiva, 5(1): 63-85. Buenos Aires: Universidad Nacional de Lanús. https://www.scielosp.org/article/ scol/2009.v5n1/63-85/

Zapata, L. y M. Genovesi (2013). Jeanne Favret- Saada: "Ser afectado" como medio de conocimiento en el trabajo de campo antropológico". Avá. Revista de Antropología, 23, 49-67. Argentina: Universidad Nacional de Misiones. 